\title{
Administration of Infrared Transillumination Method for Improving Diagnostic Outcomes of the Partial Nephrectomy
}

Sopio abazadze", Alexandre khuskivadze and Besarion Partsvania

Lecturer, Department of Pathology, New Vision University

\author{
"Correspondence author \\ Sopio Abazadze \\ Lecturer \\ Department of Pathology \\ New Vision University
}

Submitted : 27 April 2020 ; Published : 8 May 2020

\section{Abstract}

Over the past two decades, the incidence of the kidney cancer has increased by $2 \%$ worldwide. It will appear in the VI-VII decade of life (average age 60 years). Kidney cancer was previously considered to be an older person's disease, however according to the world health organization 2017; the number of young people with kidney cancer has unfortunately increased. Most of renal malignancies are so called renal-cell carcinoma (RCC) [1]. As for kidney, sarcoma and Wilms tumor are much rear.

Currently, more than $50 \%$ of cases of kidney cancer are discovered incidentally in instrumental studies for a variety of reasons. For its diagnosis is mainly used radiological studies, in particular: ultrasonography and commuter tomography (CT) with contrast. As for lab tests, investigations are curried out for exclusion of paraneoplastic syndrome. CT with contrast is most informative in kidney cancer diagnosis. It helps us to identify: 1) structure and function of the kidney, 2) probable margins of the primary tumor, 3) Evaluation of local lymph nodes.

RCC treatment is surgical removal of the tumor. Current guidelines recommend $\mathrm{PN}$ as the treatment of choice for the majority of patients diagnosed with a small renal mass Campbell et al, Ljungberg et al [1, 2]. PN provides the same oncological efficacy as radical nephrectomy, with the added benefit of minimizing the risk of chronic kidney disease [3]. PN should always be done (if technically possible) in patients with renal cell carcinoma in a solitary kidney, if bilateral tumors are present, in chronic renal insufficiency or for patients with hereditary renal cell carcinoma.

However, leaving a positive surgical margin remains one of the risks associated with nephron-sparing surgery, because this can lead to higher chances of cancer recurrence. In the course of such a partial nephrectomy, an immediate express morphological examination of the removed specimen is performed. The purpose of this express investigation is to determine whether cancer is remained in non-resected part of kidney. During the express examination, surgery is suspended and surgeon is awaiting a response of the express examination, to which the next action of the surgeon is greatly dependent. This study includes specific (so called intra-operative frozen section (FS) histo-morphological exmination. If tumor is detected by this examination at the margin, which was supposed by surgeon as healthy tissue, surgeon will be informed about incomplete removal of tumor. The implication of this finding is a potential increased risk for local or distant recurrence. Such patient is subject to special observation during the postoperative period. Which is associated with additional financial and radiation risk During PN, so called intra-operative frozen section (FS) of the removed specimen is commonly performed to confirm the presence of a negative surgical margin $[4,5]$. Sidana et al, However, results of FS is not characterized with high accuracy. By this reason, after its execution specimen is processed using routine histo-morphological technique.

It is noteworthy that the recurrence rate of cancer in patients with positive diagnosis in removed specimen was $16 \%$, whereas in the case when diagnosis give of negative answer the recurrence was only 3\% [6]. As it is well known, at the beginning of surgery, the blood vessels supplying the kidney are clamped, which for a long time is inadmissible. This can cause renal ischemia and irreversible changes of nephrons in the kidney. Ischemia time should be limited whenever possible: 20-30 min should be the expected maximum duration of warm ischemia to prevent long-term renal dysfunction [7]. 
Consequently, from the stated, as further patient surveillance and adherence to oncological principles are significantly dependent on express examination results, new alternative methods are seeking that would significantly reduce the burden of pathologist and improve the accuracy of diagnosis. For example, 7 Tesla MRI is used to examine surgical kidneys specimens [8]. The disadvantages of this method are: It is complicated, expensive and cannot be used widely in clinics. It also requires both a highly qualified radiologist as well as special trained doctor. This is especially true for countries like Georgia. In some countries launched usage of so called structural illumination microscopy [9]. However, it should be noted that this method is in the processing stage. Its drawback as well as MRI is its complexity and high price. Like MRI it is not used in Georgian clinics. As for use of ultrasound on already removed specimen, data of existing studies show that this method is not precise.

Different, much fast, safer and cheaper method than existing ones (histo- morphology, MRI and structured light microscopy), is presented developed in this work. This infrared (INR) imaging method will allow accurate determination of the margins of cancerous formations. As a result, in the case revealing positive margines the surgeon will be able to remove the additional portion and until negative margins will be achieved. Infrared Trans illumination technique has been widely used for imaging breast cancer, for prostate cancer imaging Partsvania [10-12].

\section{Methods}

Studies include the acquisition of 2D Tran's illumination images. The method allows the detection of a local cancerous area of several millimeters. In the case of examining of removed kidney specimen, infrared rays will travel in about 4-4 cm thick tissue. Experimental unit consists of infrared radiation source, holder for cancerous tissue, and CCD camera. Rays emanating from the infrared source pass through kidney tissue. Rays coming from tissue, containing the information about optical density inhomogeneity, fall into the infraredsensitive matrix. The output of the CCD camera will be connected to the computer, where developed software coverts electrical signals coming from CCD camera into visual images -IR images [12]. In the figure, 1 is shown experimental setup for IR investigations.

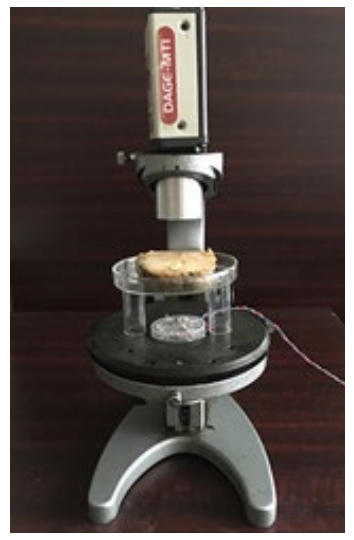

Figure 1: Experimental set up. Kidney specimen is placed on Petri dish. LEDs (shown with arrow) are placed under the specimen. CCD camera (shown also with arrow) is connected to PC. IR passing through tissue carries information about optical density inhomogeneity caused by existence of cancerous formations.

Thus, the photodiodes (LED) will be used as infrared light source, which ensure equal illumination of the study material. We will be able to regulate the intensity of LED irradiation. By doing so, we will achieve that each examined specimen will be put under the same experimental conditions. In the case if, the test tissue being uniform, the intensity of the infrared rays coming out from this tissue will be the same at all points. If the tissue is heterogeneous, for example, it contains both the cancerous formation and healthy tissue, because the optical densities of these tissues are different, the infrared light intensities coming from them will be different [12-14]. For example, because the cancerous tissue is optically denser than the noncancerous one, the intensity of the infrared light coming out from this tissue will be less than of the healthy tissue. Thus, light coming out from each point of study tissue will have its own intensity value. Method allows simulations examination of whole kidney specimen removed during surgery and requires only few minutes.

\section{Results}

Figure 2 shows the IR images of two different kidneys. On the left is given IR image of the cancerous kidney specimen, removed during the surgery. Cancerous formation is indicated by light arrow. The margins of the cancerous formation is clearly seen. IR image of noncancerous kidney is characterized by even distribution of brightness on all image. This is so because optical density of noncancerous tissue is one and the same at each points of the tissue.

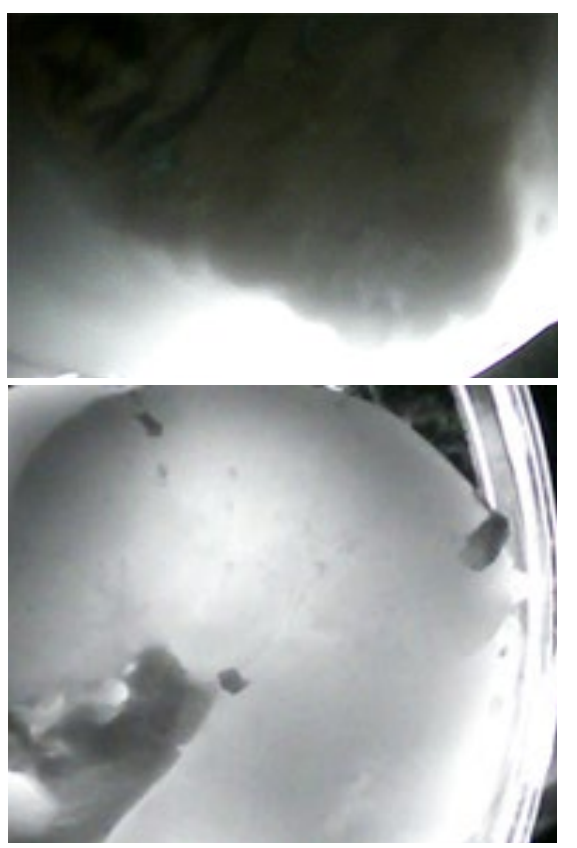


Figure 2: Infrared image of the specimen of cancerous kidney is shown on the left. Cancer margin are clearly visible. The black arrow shows tissue in a heealthy area. The light arrow indicates the canceous formation.

To the right is the infrared image of part of the noncancerous kidney (placed ion the Perty dish). Brightness of image is practically homogenneous.

After performing of statistically reliable number of experiments (at least 30 experiments for each type of kidney cancer), developed software will allow us to investigate unknown kidney tissue and determine margins of cancerous formation and healty tissue with $95 \%$ probability.

\section{Conclusion}

Administration of IR trans illumination 2D images is promising method for reliable and prompt determination of cancerous margins in the isolated kidney specimen and might substitute existing complec methods.

\section{References}

1. Campbell SC, Novick AC, Belldegrun A, Blute ML, Chow GK, et al. (2009) Guideline for management of the clinical T1 renal mass. J Urol 182: 1271-1279.

2. Ljungberg B, Cowan NC, Hanbury DC, Hora M, Kuczyk MA, et al. (2010) EAU guidelines on renal cell carcinoma: the 2010 update. Eur Urol 58: 398-406.

3. Touijer K, Jacqmin D, Kavoussi LR, Montorsi F, Patard JJ, et al. (2010) The expanding role of partial nephrectomy: a critical analysis of indications, results, and complications. Eur Urol 57: 214-222.

4. Breda A, Stepanian SV, Liao J, Lam JS, Guazzoni G, et al. (2007) Positive margins in laparoscopic partial nephrectomy in 855 cases: a multi-institutional survey from the United States and Europe. J Urol 178: 47-50.

5. Sidana A, Donovan JF, Gaitonde K (2014) Surgeons' preferences and practice patterns regarding intraoperative frozen section during partial nephrectomy. Urol Oncol 32: 864-868.

6. Wood EL, Adibi M, Qiao W, Brandt J, Zhang M, et al. (2018) Local Tumor Bed Recurrence Following Partial Nephrectomy in Patients with Small Renal Masses. J Urol 199: 393-400.

7. Thompson RH, Lane BR, Lohse CM, Leibovich BC, Fergany A, et al. (2010) Every minute counts when the renal hilum is clamped during partial nephrectomy. Eur Urol 58: 340-345.

8. Van Oostenbrugge T, Runneboom W, Bekers E, Heidkamp J, Langenhuijsen JF, et al. (2019) MRI as a tool to assess surgical margins and pseudocapsule features directly following partial nephrectomy for small renal masses Eur Radiol 29: 509-516.

9. Wang Mei, Tulman David B, Sholl Andrew B, Mandava Sree H, Michael M Maddox, et al. (2018) Partial nephrectomy margin imaging using structured illumination microscopy. J Biophotonics 11.

10. Fantini S, A Sassaroli (2012) Near-Infrared Optical Mammography for Breast Cancer Detection with Intrinsic Contrast. Ann Biomed Eng 40: 398-407.

11. Partsvania (2016) Near infrared transillumination detection of prostate carcinoma in vitro. Journal of Cancer Science \& Therapy 8.

12. Partsvania, Petriashvili G, Fonjavidze N (2014) Possibility of Using Near Infrared Irradiation for Early Cancer Diagnosis. Electromagnetic Biology and Medicine 33: 18-20.

13. Besarion Partsvania, Tamaz Sulaberidze, Alexandre Khuskivadze, Levan Shoshiashvili, Ketevan Chubinidze (2016) Infrared light enables visualization of the prostate carcinoma after radical prostatectomy. Oncology Discovery 4: 1-5.

14. Thompson R Houston, Blute Michael L (2007) At What Point Does Warm Ischemia Cause Permanent Renal Damage during Partial Nephrectomy? European urology 52: 961-963.
Copyright: (C2020 Sopio abazadze. This is an open-access article distributed under the terms of the Creative Commons Attribution License, which permits unrestricted use, distribution, and reproduction in any medium, provided the original author and source are credited. 